\title{
Enquête
}

Archives de la revue Enquête

4 | 1996

La ville des sciences sociales

\section{Conceptions, structures matérielles, pratiques}

Réflexions autour du « projet urbain »

Conceptions, material structures, practices. Reflections on the "urban project"

\section{Marcel Roncayolo}

\section{(2) OpenEdition}

Journals

Édition électronique

URL : http://journals.openedition.org/enquete/703

DOI : 10.4000/enquete.703

ISSN : 1953-809X

Éditeur :

Cercom, Éditions Parenthèses

Édition imprimée

Date de publication : 1 novembre 1996

Pagination : 59-68

Référence électronique

Marcel Roncayolo, «Conceptions, structures matérielles, pratiques », Enquête [En ligne], 4 | 1996, mis en ligne le 11 juillet 2013, consulté le 20 avril 2019. URL : http://journals.openedition.org/enquete/703 ; DOI : 10.4000/enquete.703

Ce document a été généré automatiquement le 20 avril 2019. 


\title{
Conceptions, structures matérielles, pratiques
}

\author{
Réflexions autour du « projet urbain » \\ Conceptions, material structures, practices. Reflections on the "urban project"
}

\section{Marcel Roncayolo}

1 Chercheurs des sciences sociales, urbanistes et architectes ont construit des objets qui se révèlent peu perméables les uns aux autres. S'il nous faut rétablir non pas des objets unifiés d'une discipline à l'autre mais la possibilité d'une rencontre et d'une confrontation, il s'agit moins d'un travail institutionnel qu'intellectuel. Nous nous interrogeons tous sur la nature et la notion du projet urbain. D'une part parce que ce mot exprime l'un des aspects fondamentaux que l'on prête à l'architecture et à l'urbanisme, l'idée de conception et de projection; d'autre part, parce que la mise à l'ordre du jour du "projet urbain» correspond à une inflexion précise de la pensée urbaine et de la définition de l'action en ce domaine, à une conjoncture qui s'est dessinée dans les années soixante-dix. Et nous sommes souvent amenés à balancer entre ces deux références ${ }^{1}$.

2 L'histoire à long terme du projet reste dans l'incertitude, tiraillée entre les risques d'anachronisme et ceux de ruptures excessives, et éventuellement mal situées, dans les périodisations. Les oppositions trop souvent rhétoriques entre villes de fondation et villes de sédimentation ${ }^{2}$, conceptions et conventions, cités idéales et réalisations nous laissent dans le flou sur les démarches réelles. Il serait sans doute souhaitable de bâtir une histoire de l'intervention (et même du volontarisme) en matière urbaine, en identifiant mieux les pratiques, les vocabulaires, la division des compétences, des métiers et des interprétations. C'est donc par prudence que l'on évoque moins les origines que la comparaison avec des expériences plus proches de nous : pratiques et idéologies du temps des Lumières, mise en place juridique des plans d'extension et des plans régulateurs, création de quartiers du premier xixe siècle - le quartier de l'Europe, par exemple, à Paris - et les différents modes d'intervention liés à l'haussmannisation (par exemple création de quartier et percée à Marseille, autour des terrains de la Joliette) ${ }^{3}$. 
3 Dans sa version conjoncturelle plus actuelle, le terme de "projet urbain » me parait correspondre, à la fois comme expression critique et substitut, aux formes de planification qui ont marqué la grande période de croissance et d'extension autour des années soixante, et qui se caractérisaient à la fois par le schématisme, la fragmentation, et la juxtaposition de logiques sectorielles. On s'efforçait d'inviter à de nouvelles rationalités, moins détachées des hommes, plus proches des conduites et des aspirations, surtout plus complexes et retraçant, somme toute, au-delà des coupures sectorielles, l'unité des territoires. "Retour à la ville ", "Retour à l'architecture " résumaient en grande partie l'intention - tout en soulignant les questions que cette inflexion soulèverait. Car proclamer le projet « urbain », était-ce simplement une affaire d'échelle? ou une différence dans le contenu même du projet et par conséquent dans sa conception, les compétences à réunir, les négociations à mener, les temporalités à maîtriser ?

4 À travers cette double dénomination de " projet » et d'« urbain », la notion n'a jamais été totalement éclaircie, même si les points de vue se sont affrontés. Ce n'est point forcément un reproche: une notion qui n'est éclaircie ni trop tôt, ni trop mécaniquement, quand elle vient en contre-feu de pratiques antérieures, est plus productive d'expériences et de réflexions que lorsqu'elle se fige et entre dans le carcan de procédures imposées. L'intérêt de la notion de "projet urbain", même si elle a subi des fluctuations de mode, c'est précisément cette sorte de labilité qu'elle introduit dans la manière de concevoir la construction des villes et l'aménagement ou la redéfinition de certains espaces hérités. Le risque, c'est que la notion et le mot ne deviennent qu'un pavillon couvrant la marchandise, une marchandise qui reste grosso modo la même. Le projet urbain (comme le projet de quartier invoqué à plusieurs reprises) veut alors n'être qu'un avatar de la pratique du zonage. C'est pour cela qu'il faut aller plus loin dans l'analyse. Je reviendrai donc sur la question des échelles et sur une notion connexe du projet urbain, qui est celle de contexte.

\section{Échelles et contextes}

5 Échelles? Les sciences humaines sont en grande partie redevables sur ce point, de la cartographie et de l'architecture ${ }^{4}$. Il est clair que les échelles sont des phénomènes de représentation, répondant évidemment à la nature des phénomènes mais surtout commandant des modes d'études différents. Urbain et architectural paraissent se situer à deux niveaux de la réalité et comporter des caractères spécifiques : il ne s'agit pas, par conséquent, d'un simple jeu de poupées gigognes! Ville et édifice, dont, à juste titre, Christian Devillers ${ }^{5}$ rappelle qu'ils appartiennent à des temporalités très différentes, long terme pour la première, terme plus court pour le second. Toutefois le projet urbain d'aujourd'hui ne peut guère être identifié à une forme globale de la ville qui paraît se perdre dans une expansion et une mobilité plus généralisées et dépendre, somme toute, de l'organisation des réseaux plus que d'un dessin visible, distinctif. C'est donc à des échelles intermédiaires que l'« urbain "se définirait à l'égard de l'architectural et l'on comprend que le projet urbain ait été rapproché de la composition urbaine.

6 La notion de projet urbain n'est plus appliquée à l'ensemble de la ville et c'est sur ce point, d'abord, que l'architecte se proclame modeste. Le souci de traiter des "morceaux " de ville sort renforcé de ce retour aux réalités du paysage urbain et des dimensions de sa fabrication. Il ne s'agit pas d'un refus d'intervenir ou de planifier, mais d'une méfiance à l'égard d'une programmation trop systématique, abstraite, qui aboutit à substituer les 
procédures au modelage plus fin de l'espace urbain. On cherche en fait à se rapprocher du terrain, du site, des acquis de l'usage, des pratiques des habitants. Le mot « morceau » luimême peut être inquiétant, s'il ne signifie qu'un nouveau découpage du territoire et la clôture des opérations sur elles-mêmes ; sans doute faudrait-il rapprocher le sens plutôt de son emploi musical qui n'exclut ni les enchaînements ni les séquences.

Cette vision du " projet urbain » me parait loin de toucher à son terme, à l'heure actuelle, même s'il convient d'en noter les limites, les échecs ou le caractère peu explicite. Les applications ne sont pas seulement affaire d'étendue (grands projets ou petits projets), d'ambition, de conjoncture (une idéologie pour croissance ralentie), elle touche la manière de concevoir la relation avec la ville héritée, la médiation entre les échelles, le rapport tout à la fois avec les disciplines et les parties prenantes qui ont quelques compétences dans la définition et éventuellement l'exécution d'un projet urbain. Ce que j'appellerais volontiers le contexte.

8 Contexte spatial ${ }^{6}$, d'abord: on sait que la notion de projet urbain s'inscrit en grande partie dans l'inflexion intellectuelle de l'architecture autour de la typo-morphologie - et donc de la révision entreprise par les architectes italiens voici une génération. La découverte à la fois des logiques des formes urbaines, de la relation entre les échelles et de l'importance des trames acquises n'est plus à rappeler. Dès lors, qu'il s'agisse de requalifier d'anciens tissus, d'utiliser la gamme des «praxis » existant dans la ville, de penser à rebours le lien du modernisme en termes de continuité et non de rupture, l'analyse des formes urbaines devient essentielle, condition dans une certaine mesure de la conception. En même temps les formes ne peuvent être situées uniquement à leur échelle mais rattachées, au moins intellectuellement, par enchainement, à d'autres formes acquises ou projetées : mises en perspective, ouvertes aussi. De ce point de vue, le projet urbain ne peut être jugé exclusivement sur les expériences entreprises et réalisées: ce qui reste plus important, c'est qu'il introduit (nécessairement, faut-il l'espérer) une réflexion sur le temps et la maîtrise du temps. Ce qui rejoint mes plaidoyers de naguère, un peu provocateurs, pour que la géographie soit considérée comme une discipline du temps et pas seulement de l'espace, peut-être plus du temps que de l'espace.

Le contexte, toutefois, ne peut s'arrêter à cette sorte de cartographie en mouvement de l'inscription sur le sol ou de la lecture en volume ${ }^{7}$. On peut toujours reconnaître l'inertie, la résistance, l'identité de paysage venant de la morphologie matérielle de la ville, par exemple du jeu des voies, du parcellaire et du bâti. On ne peut oublier que les uns et les autres répondent à des exigences ou à des essais qui sont de nature sociale. Et ce, quelle que soit leur labilité par rapport aux usages initiaux. Ce sont toujours des " aménagements » - construits volontairement ou provenant d'une lente sédimentation, d'un ajustement par des acteurs anonymes, selon une chronologie peu définissable. La leçon des paysages agraires est là pour nous conforter. L'ajustement des techniques, les répartitions sociales du travail, du pouvoir ou même les représentations s'établirent toujours entre formes et sociétés. La limite de la typo-morphologie ne vient pas de défaillances méthodologiques, de sa vertu descriptive (y compris à travers les successions historiques) plus qu'explicative que cette question centrale: le rapport entre formes matérielles et formes sociales. Question d'autant moins aisée que la relation n'est ni immédiate, ni univoque, que les usages, les pratiques, les sens peuvent, comme les formes ou indépendamment des formes, changer. Un autre contexte doit être alors défini, dans l'analyse assurément, dans le travail de projection aussi, avec des compétences qui relèvent des sciences humaines et sociales. Ce qui ne signifie pas que des architectes 
soient incapables de les assimiler, de même que la morphologie matérielle ne me semble pas chasse-gardée. En ce domaine, je crois plus aux compétences définies par le métier et la pratique, l'apprentissage aussi, qu'aux bannières intellectuelles.

\section{La conception et le processus social}

10 Cela ne veut pas dire qu'il ne faut pas prendre en compte la conception. J'ai beau venir de la discipline la plus tournée vers les aménagements matériels, la géographie, j’attache le plus grand prix à cette dimension, y compris au rôle de l'artiste en tant que tel. La conception me paraît avoir été victime, en quelque sorte, de la planification spatiale telle qu'on la concevait dans les années soixante. Le projet urbain, dans sa forme « idéale » me paraît répliquer à cette défaillance, avec plus ou moins d'efficacité. À travers une opération que l'on pense surtout comme individuelle, une complexité de relations apparait aussi - dans les ébauches, les projets restés à l'état de papier ou de dessin, ou ceux qui parviennent à l'étape de l'exécution. La conception repose sur une certaine maîtrise intellectuelle d'une série d'éléments, un certain rapport que l'on établit entre les contraintes de l'espace et de la fabrication et le destin social (usage personnel, espace public, signification collective) de l'objet ou de l'ensemble pensé. Il n'y a pas, je pense, de conception sans ces deux références, donc sans un certain finalisme. Il se trouve que l'architecture (à l'échelle de l'édifice comme à celle des compositions de la ville) n'est pas dissociable d'un certain dessein social - et ce n'est pas une caractéristique propre au modernisme ou à ces excès. Or l'espace est peut-être plus limité que le rêve social. Dans cette perspective, la création me semble balancer entre la reproduction et l'anticipation. La reproduction offre certes des garanties mais aussi une issue un peu négative par rapport à une histoire qui n'est guère faite d'équilibres stables. L'anticipation implique bien plus qu'une innovation technique ou formelle; elle n'est pas seulement projection de règles nouvelles qui répondent à un moment déterminé, mais essai d'envisager un avenir ou, éventuellement, des avenirs multiples. De ce fait, l'anticipation ne peut être exclusivement formelle ou même économique: elle touche nécessairement à la transformation de la culture et plus précisément des pratiques. La conception relève donc d'un type d'histoire qui ne peut isoler l'acte de construction des autres manifestations culturelles d'une époque dans la manière de se penser elle-même ou de se projeter dans l'avenir. Même si Francastel ${ }^{8}$ simplifiait en lisant dans la ville des strates superposées, à la manière géologique, et donc utilisait à l'excès l'image du palimpseste, il ne se trompait guère en situant les transformations de Paris vers le second Empire dans ce contexte. Mais on voit les risques que comporte l'anticipation non critique sur les besoins et les conduites. « Construire pour vingt ans » manifestait, au lendemain de la dernière guerre, une bonne conscience un peu facile justifiée par l'urgence ou la croyance dans un progrès accéléré. On connaît la suite.

11 Ce qui me semble faire actuellement défaut, c'est de considérer la conception elle-même comme un processus et plus précisément un processus social, qui implique des individus, des groupes, des "décideurs", au sein d'une société moins cohérente qu'on ne le pense. Conception processus, assurément. Mais la conception s'établit aussi comme une étape dans une série de processus qui vont de la construction intellectuelle du projet à sa réalisation, à sa façon de prendre place parmi les pratiques et l'évolution des usages. La sociologie de l'appropriation a porté, sur ce point, des regards fort pertinents. Si les projets architecturaux et urbains, les dessins des villes ou les projections graphiques 
fournissent une matière très riche dans l'analyse culturelle d'une époque, qu'ils soient accueillis ou rejetés, ils ne donnent qu'une image tronquée de la série de processus dans laquelle l'œuvre architecturale et urbaine se trouve prise. L'histoire de l'art et celle du patrimoine mesurent aujourd'hui ce changement.

Il ne s'agit pas de vouloir préserver la liberté de l'homme, de l'individu ou des collectivités, mais plutôt d'éviter les faux problèmes qui nous feraient en permanence opposer la conception à ce qui serait déviation, ratage ou trahison. Ce que l'histoire ou la géographie historique nous enseigne se résume aisément : les usages et plus précisément l'enchaînement des usages dans le temps sont rarement conformes à l'idée initiale, même s'il s'agit d'un processus complexe d'élaboration. Parler de processus, c'est parler d'acteurs sociaux (de tous ordres) et de leur succession dans le temps.

\section{Multi-temporalités urbaines}

13 Dès lors qu'il y a création, remodelage, requalification d'espace urbain, il y a nécessairement négociation. Il est d'autre part impossible d'analyser ou de comprendre les conditions de cette négociation sans redonner toute son importance à la notion de temps ${ }^{9}$, ce qui implique de la repenser avec fermeté. Nous jouons, dans nos analyses comme dans nos projections, sur des temps très variés, que nous contrôlons intellectuellement de manière très inégale. Si l'on est prêt à conserver au projet urbain son caractère complexe, synthétique (par rapport, par exemple, aux découpages logiques en réseaux), en perspective, il faut admettre que le temps dissocie, dans leur déroulement, les composantes du projet urbain, qu'il en distingue à la fois les étapes et les épisodes. Temps de la création, de la négociation, des usages ne sont pas les mêmes, non seulement par le fait qu'ils se succèdent ou se chevauchent inégalement, mais parce que les actions et les objets sur lesquels ils portent obéissent à des temporalités très différentes.

Reprenons des exemples dans l'histoire urbaine. L'étude de l'haussmannisation sert aussi à cela. Dans la relation entre l'exécution et le financement, la tension apparaît constamment, d'autant plus que les grands spéculateurs qui interviennent n'obtiennent de crédit qu'à la mesure des bénéfices espérés. Les grandes compagnies se livrent donc à une série de manœuvres pour gonfler leurs résultats initiaux qui ne peuvent être maintenus, ni honorés quand vient le temps des réalisations sérieuses. Marseille en fournit un excellent exemple avec sa rue Impériale. L'haussmannisation met aussi en évidence des temporalités différentes ${ }^{10}$. Lisons Maurice Halbwachs: la valeur des emplacements atteint rapidement son meilleur niveau dans les emplacements centraux, là où les transformations entreprises à partir $\mathrm{du}$ second empire trouvaient immédiatement leur justification et leur emploi. Au contraire, à la périphérie - malgré même le caractère social de l'occupation du sol -, les prix ne haussent fortement qu'au terme d'un certain délai, de dix ou vingt ans. M. Halbwachs évoque le cas de l'avenue Henri Martin, dans le $16^{\mathrm{e}}$ arrondissement de Paris: "L'expropriation est loin d'avoir élevé les prix immédiatement; il a fallu près de vingt ans et des percées nouvelles à proximité pour qu'on y voie le commencement d'une hausse des prix du terrain. Elle est un signe très net de la lente expansion qui a porté vers l'ouest extrême la population riche de Paris ${ }^{11}$." Déplacements qui ne sont pas simplement l'effet d'une évolution quantitative mais de choix socioculturels. Temps d'anticipation et de maturation n'ont pas les mêmes phases. 

matérielle des villes; voies, parcellaire, bâti paraissent appartenir à un même système et donc exprimer les attentes d'une même époque. Mais la dissociation, sous certaines conditions, a des chances de s'opérer. La trame résiste le mieux même quand elle ne répond plus tout à fait aux exigences de la modernité. Le parcellaire n'est pas une donnée figée, même si les tracés généraux persistent : les parcelles sont agrégées, regroupées ou subdivisées et changent d'usage. Les édifices subissent davantage les modifications, transformations, processus de démolition/reconstruction, au gré des calculs du marché et des stratégies patrimoniales. Évoquons encore l'exemple de Marseille ${ }^{12}$. La trame orthogonale de la nouvelle ville, tracée et construite au XVII ${ }^{\mathrm{e}}-\mathrm{XVIII}{ }^{\mathrm{e}}$ siècle, subsiste à peu de chose près et a même engagé, par continuité, le dessin des zones de croissance qui se sont greffées sur elle. Mais là où les constructions n'étaient ni trop denses ni trop serrées, là où le dessin des parcelles laissait du jeu, les édifices ont été repris, reconstruits, réaffectés en fonction d'activités propres à la centralité, quelquefois deux à trois séries d'immeubles se substituant les uns aux autres, quitte à entrer, en certains lieux, dans des enchaînements de déqualification dans les proches décennies. Si nous faisons un saut à New York, nous passons dans la trame projetée en 1811, la "grille ", à partir d'îlots à peine modifiés et sur un parcellaire le plus souvent regroupé, de la première génération de maisons classiques à trois étages aux buildings du postmodernisme.

Les structures matérielles de la ville ont des rythmes de vie variables, dans lesquels jouent non seulement les rationalités des époques de création, revues, réinterprétées, corrigées, mais aussi les intérêts qui tiennent à leurs propriétaires, à leur affectation, parfois à la valeur symbolique. Elles deviennent des enjeux, le cas échéant dissociables. Ainsi, à la pérennité de la ville peut-on opposer les processus de démolitionreconstruction, déqualification-requalification (sans donner de valeur positive nécessairement à l'une ou à l'autre), et les changements de sens. Les groupes d'intérêts (au sens le plus large du terme), les groupes sociaux eux-mêmes et leur mémoire sont loin d'être alignés et d'obéir aux mêmes temporalités.

algré les tendances plus ou moins fortes, portées à l'unification (la mise à l'heure de la modernité ou du temps social global), les temps sont divers, dans leur origine, leur déroulement, leurs phases : c'est leur articulation, éventuellement leur confrontation qui fait la ville, si bien qu'à la limite elle peut apparaître à l'opposé de la synchronie. La compréhension de ces non-synchronies ou de ces a-synchronies, de ces alignements difficiles à un rythme organisateur, est essentielle à l'analyse et à la conception du projet urbain ; quelle que soit son ampleur, il n'est jamais destiné à rester tel qu'en lui-même l'architecte ou l'urbaniste l'aurait pensé. L'incertitude plane encore plus aujourd'hui sur tout ce qui concerne les proximités physiques et les distances temporelles, dont on sait les variations et les caractères de moins en moins commensurables. Ce qui est essentiel pour identifier les cohérences ou les non-cohérences urbaines.

tes banlieues ou les zones suburbaines qui auraient rendu la démonstration encore plus aisée, sous réserve des différences entre pays et cultures. Mais c'est aussi là que les notions d'enchaînements, de séquences, d'articulations (à travers les espaces publics) s'imposent à une autre échelle sans doute. L'urgence de coutures y entre peut-être en contradiction avec le maintien de cette labilité, de cette perspective « ouverte » dont notre temps incertain exprime l'exigence.

Le véritable problème me semble résider dans la relation entre les différentes manières de concevoir le temps. D'une part, le temps des créateurs, des architectes, des urbanistes, 
des ingénieurs - celui des projections. D'autre part le temps des sciences sociales qui, en dehors des grandes utopies, restent accrochées au présent et à une approche des tendances le plus souvent rétroactive - en tout cas qui cède peu au mirage de la divination. Le temps enfin des ajustements entre formes et société, dont les croisements échappent, en fin de compte, à nos déterminismes et à notre prévisibilité pour partie et peut-être pour l'essentiel. Pour éviter l'éclatement entre ces temps, en saisir au contraire quelques articulations, il faut sans doute les concevoir, non comme une opposition radicale entre le passé et le futur, de part et d'autre d'un présent, qui paradoxalement, reste qualifié par une durée incertaine, mais plutôt comme le développement d'actions, de trajectoires entre passé, présent et futur. C'est cette logique-là qu'il faut retrouver. Elle permet d'atténuer les distances entre les détenteurs des sciences de l'analyse et les domaines de la projection; dominer, dans la mesure du possible cette multiplicité des temps permet de retrouver le minimum de cohérence intellectuelle qu'exigent, sous ses différentes formes et dimensions, le projet urbain et la réflexion sur l'urbain tout court. C'est la rencontre des disciplines qui est alors en jeu, non pas sous leur aspect purement institutionnel, mais à travers leurs pratiques, la formation et les aptitudes, les modes de raisonnement, le substrat culturel de leurs représentants. Pour ce qui concerne le projet urbain, il s'agit non d'abolir des différences, mais de réussir à constituer des unités de travail capables d'intégrer, partiellement ou totalement, les apports spécifiques.

\section{NOTES}

1. Ces réflexions reprennent une intervention présentée à l'école d'architecture de Marseille le 31 janvier 1997. Sur le projet urbain, je renvoie à quelques textes nés des débats entre architectes et urbanistes: C. Devillers, Le projet urbain, Paris, Pavillon de l'Arsenal, 1994 ; B. Haumont, «Un nouveau champ pour l'architecture et ses recherches : le projet urbain », Cahiers de la Recherche architecturale, 32-33, 1993, p.103-110; P. Genestier, "Que vaut la notion de projet urbain", L'architecture aujourd'hui, 288, septembre 1993; Direction de l'Architecture et de l'Urbanisme, Comprendre, penser, construire la ville. La communication de Patrizia Ingallina au colloque de Barcelone «Ville et sémiotique ", juin 1996, m'a introduit à une «approche comparée francoitalienne » de la question du projet urbain.

2. P. Lavedan, Qu'est-ce que l'urbanisme?, Paris, H. Laurens, 1926, a développé cette analyse parmi les premiers.

3. Histoire de la France urbaine, Paris, Seuil, t. III et IV, 1981-1983.

4. B. Lepetit, "Architecture, géographie, histoire: usages de l'échelle ", Genèses, 13, 1993, p. 118-138.

5. C. Devillers, op. cit., p. 30, et "De la logique de secteur au projet urbain », Villes en parallèle, 12-13, 1988, p. 244 sq.

6. Critique, le livre collectif dirigé par P. Merlin et F. Choay traite notamment des rapports entre morphologie urbaine et projet. Morphologie urbaine et parcellaire, colloque d'Arc-et-Senans, 28-29 octobre 1985, Saint-Denis, Presses universitaires de Vincennes, 1988. Voir également Villes en parallèle, 12-13, 1988, sur les « Formes urbaines ».

7. M. Roncayolo, «La morphologie entre la matière et le social », entretien avec G. Burgel et P. Genestier, Villes en parallèle, op. cit. 
8. P. Francastel, «Paris, un héritage culturel et fondamental », in Notes et Études documentaires, Paris, La Documentation française, 1968.

9. Cf. Temporalités urbaines, B. Lepetit, D. Pumain, eds, Paris, Anthropos, 1993, en particulier l'article de B. Lepetit, p. 113-134.

10. Je renvoie à la conclusion des Grammaires d'une ville. Essai sur la genèse des structures urbaines à Marseille, Paris, Éd. de l'EHESS, 1996.

11. M. Halbwachs, Les expropriations et le prix des terrains à Paris, 1860-1900, Paris, 1909, p. 363.

12. M. Roncayolo, Les grammaires d'une ville, op. cit.

\section{RÉSUMÉS}

À la croisée des sciences sociales, de l'urbanisme et de l'architecture, la notion de "projet urbain » est apparue comme en opposition aux formes de la planification, à l'œuvre durant la grande période de croissance et d'extension des villes autour des années soixante. Malgré l'indétermination qui l'affecte, elle marque au moins une double rupture: expression d'une méfiance face à une programmation systématique, elle situe l'intervention près du terrain, au niveau de "morceaux de ville»; ces «morceaux » ne sont pas isolables, mais sont rattachés à d'autres formes, acquises ou projetées. Les difficultés, voire les échecs du "projet urbain» obligent désormais à reprendre l'analyse de la conception d'un projet comme un processus social. Ils débouchent sur une reformulation des temporalités urbaines, caractérisées, paradoxalement, par des phénomènes d'a-synchronie, d'alignements difficiles à un rythme organisateur.

At the cross-roads of social sciences, urbanism and architecture, the notion of the "urban project" has appeared as in opposition to forms of planification implemented during the high period of growth and extension of cities around the sixties. Despite the irresoluteness which affects it, the "urban project" marks at least a dual rupture: it is the expression of a mistrust towards a systematic programation, it locates intervention near the field, at the level of the "piece of the city"; these "pieces" are not isolated but attached to other acquired or projected forms. The difficulties, or failures of the "urban project" nevertheless demand a reappraisal of the analysis of the project's conception as a social process. They result in a reformulation of urban temporalities, characterised paradoxically by phenomenon of an absence of synchrony, of difficult alignments to an organisatory rythm.

\section{AUTEUR}

\section{MARCEL RONCAYOLO}

Marcel Roncayolo (Université de Paris X Nanterre-EHESS) a conduit de nombreux travaux sur l'histoire urbaine de la France et Pépistémologie de la ville et s'est attaché en particulier aux relations entre formes urbaines et sociétés. Il a dirigé le tome 5 de l'Histoire de la France urbaine (La ville aujourd'hui : Croissance urbaine et crise du citadin, Paris, Seuil, 1985) et participé au volume L'espace français de l'Histoire de la France, sous la direction d'A. Burguière et J. Revel, Paris, Seuil, 1989. Il a récemment publié : La ville et ses territoires, Paris, Gallimard, 1990 ; L'imaginaire de Marseille, port, ville, pôle, Marseille, Chambre de Commerce et d'Industrie, 1990 ; Les Grammaires 
d'une ville: Essai sur la genèse des structures urbaines à Marseille, Paris, Editions de l'EHESS, 1996 ; Marseille, les territoires du temps, Paris, Editions locales de France, 1996. 